\title{
Application of Cartoon Like Effects to Actual Images
}

\author{
Chinmay Joshi, Devendra Jaiswal, Akshata Patil \\ Department of Organization: Information Technology, \\ Kc College of Engineering Management Studies and Research, Thane, Maharashtra, India
}

\begin{abstract}
How to cite this paper: Chinmay Joshi | Devendra Jaiswal | Akshata Patil "Application of Cartoon Like Effects to Actual Images" Published in International Journal of Trend in Scientific Research and Development (ijtsrd), ISSN: 2456-

6470, Volume-3 |

Issue-3, April 2019, pp.598-603, URL: http://www.ijtsrd.co $\mathrm{m} /$ papers/ijtsrd229 28.pdf

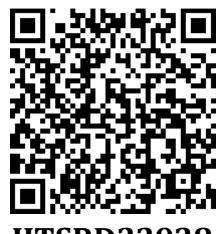
IITSRD22928
\end{abstract}

Copyright (C) 2019 by author(s) and International Journal of Trend in Scientific Research and Development Journal. This is an Open Access article distributed under the terms of the Creative Commons

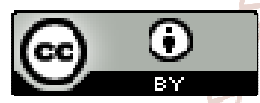
Attribution License (CC BY 4.0) (http://creativecommons.org/licenses/ by $/ 4.0$ )

\section{INTRODUCTION}

Social media is extensively used these days. And standing out in this online crowd has always been a to-do on every user's list on these social media platforms. Be it images, blog posts, artwork, tweets, memes, opinions and what not being used to seek attention of followers or friends to create influence or to connect with them on such social platforms. We aim to provide one such creative solution to their needs, which is applying cartoon like effects to their images. Users can later share these images on any social media platforms, messengers, keep it for themselves, share it with loved ones or do whatever they like with it. Nowadays almost everyone is registered in social networks. We keep online status updated every day, share photos and comments, follow our friends' news. To have a nice profile is a matter of prestige. You can use a photo of your own in a profile image, create an amusing avatar or turn your photo into a cartoon. With a pool of web applications available online, an image conversion to cartoon takes few clicks.

\section{Need of Project}

Creating a cartoon like effect is time and space consuming. Existing solutions to provide cartoon like effect to images are complex. Some solutions involve installing complex photo editing software like photoshop and other involve performing some task by user. Our research shows a website to carry out the task of Applying effects is more suitable, space efficient and takes minimum user efforts, for example toony photos is an existing website to perform such task but it is difficult to use as user has to markdown points \& lines on the image to apply effects which is not user friendly also the options are limited. Hence there is a dire need for a website which is user friendly and performs the task of applying effects to images very well.

Following is our brief research on existing solutions:

\section{A. Cartoon Effect}

The majority of photo editing websites offer the so-called Cartoon Effect. The main advantages of online photo to cartoon effect apps are simplicity and quickness. You'll have to upload a photo from your computer or from the web, find Cartoon Effect in the tool set or choose between styles or variants of this funny photo effect (like in case of www.picturetopeople.org, Kuso Cartoon ) and press the button Apply (or Go). The image processing varies from several seconds up to 1-2 minutes.

However, as all quick online solutions these apps have drawbacks. A lot of photo online photo editing tools are rather humdrum because they are deprived of enhancement features. In these apps cartoonization is limited to 1-click operation. Besides, sometimes colors may become blurred and it leads to an unsatisfactory result. Such apps as www.converttocartoon.com, Photo.to, AnyMaking and others belong to this group. At the same time there are online photo editors with more advanced tools. They have a variety of adjustment options. For example, BeFunky helps 
you modify sketch brightness, contrast, smoothness and other details.

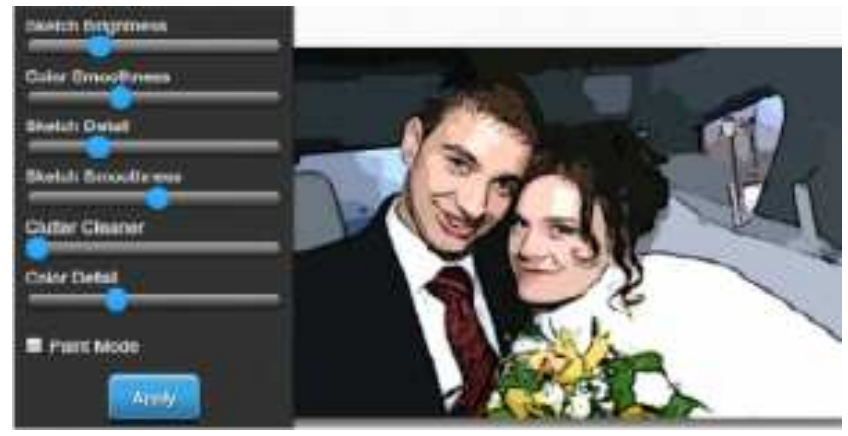

\section{B. Pencil Sketch}

Another means of cartoonization is making a pencil sketch out of your digital photograph. Whenever you apply Cartoon effect your images turn bright and cheerful. If you want to render a solid atmosphere and achieve respectability in your online profile pencil sketch creation will suit your needs better. The image manipulation procedure is just the same as described for the cartoon effect. Upload a photo, select the desired effect, push the button Apply and you are done. The application does its job instantly by itself. PhotoSketcher, Fotosketcher, Dumpr Tuxpi photo editor and many other applications give you an opportunity to convert your snaps to life-like pencil sketches.

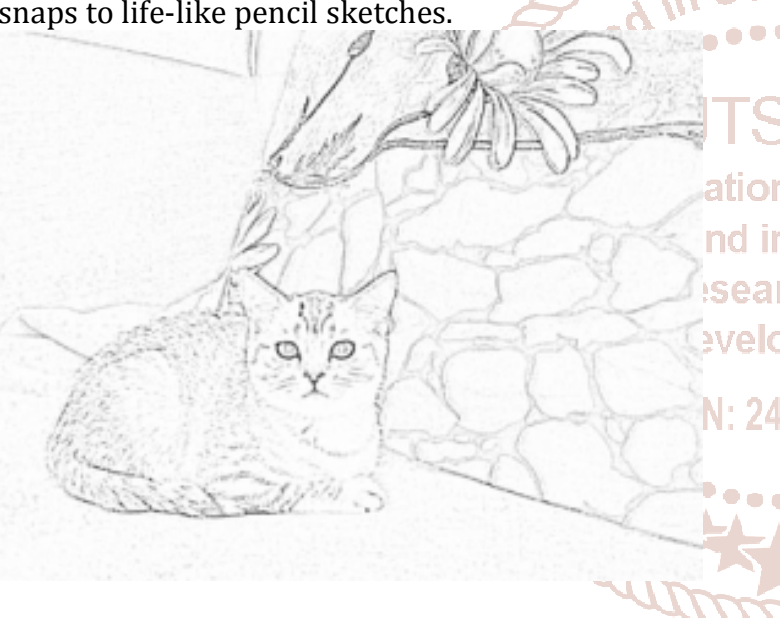

Besides, you can decorate your profile photo with a cute photo frame and even create a photo with your favorite cartoon character. Amaze your nearest and dearest, friends and coworkers with a cool profile photo, stand out from the crowd and attract more followers and fans in social networks. You know, the first impression is the strongest)). How to turn photo into cartoon online or on Windows/Mac Moreover, sharing a photo cartoon on social media could attract more attention when others just post standard photos. We are going to share how to turn photo to cartoon on Windows, Mac, and online in this tutorial. With these photo editors and our guides, you can create cartoon at any time, even if you have not learnt any knowledge about painting. If you are ready, let's start right now.

The following are the steps to convert photo to cartoon in windows MAC: -

Method 1: Convert Photo into Cartoon Online

Many people prefer to use online photo editors. They are compatible to more platforms and allow you to edit photos at anytime and anywhere. There are lots of online cartoon photo editor on the internet, you can choose one of them to make your photo into cartoon.
Cartoon a Picture Online on Toonyphotos.com

Step1. Enter the toonyphotos.com on the browser and you can see the introduction of its photos to cartoons editing function.

Step2. Click Start Turning Photos into Cartoons button to enter the cartoon photo editing webpage and then click Choose Photo button to choose the photo from your computer. After uploading the photo, you start to outline the region you need to turn into cartoon. Stop outlining by clicking the right mouse button. Click Render to start cartoonize your photo.

Step3. After applying, you can click Download to directly download the cartoon photo or click View to preview the photo before downloading. If you are not satisfied with the photo, you may need to outline more region to get more detail cartoonized photo.

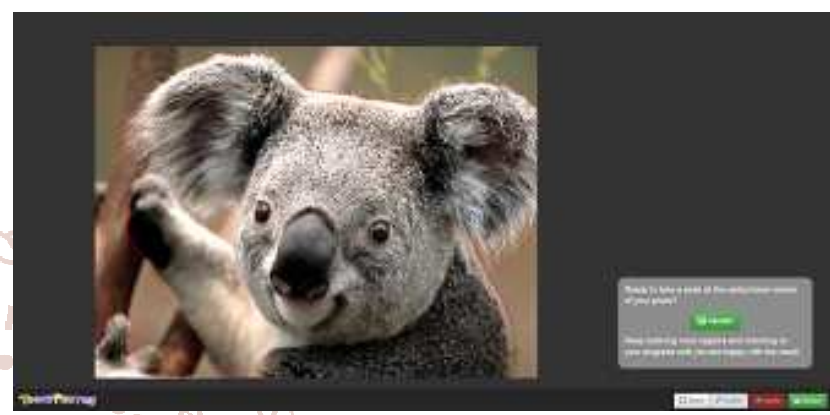

Convert photo into cartoon with Photoshop

If you have Photoshop, you can also use it to turn photo into cartoon effect in your Windows $10 / 8 / 7$ or Mac (macOS High Sierra included). The photo-to-cartoon effect in Photoshop is a popular effect and surprisingly easy to achieve. Here in this tutorial, you can learn to turn photos to cartoons using an assortment of filters and simple brushwork.

Step1. Open Photoshop and add your photo into the program.

Step2. Press Cmd/Ctrl+Shift+Alt+E to merge a new layer, then $\mathrm{Cmd} / \mathrm{Ctrl}+\mathrm{J}$ to copy it. Rename the layer Sketch.

Step3. Make a new layer, drag it below the sketch layer, then go to Edit>Fill Layer. Set Use to White and click OK. Next highlight the sketch layer and click the Add Layer Mask icon in the Layers Panel. Grab the Brush tool and set color to black, then paint to tidy up the skin, clothes and hair.

Step4. Change the Blend Mode of the sketch layer to Multiply, then make a new layer and drag it below. Grab the Brush tool and choose a color for the skin, then begin painting. Make more new layers and paint different colors for the hair, eyes, mouth and jeans.

Step5. Duplicate the background layer. Drag it to below the sketch layer. Go to Filter $>$ Brush Strokes $>$ Ink Outlines. Leave the default settings and hit OK. Altclick the Add Layer Mask icon to add a mask that hides the layer, then paint with white to reveal the tattoos. Next highlight the white layer.

Step6. Go to Filter $>$ Textures $>$ Grain. Set Grain Type: Vertical, Intensity 100, Contrast 0 . Go to Filter $>$ Blur $>$ Gaussian Blur. Set Radius $4 \mathrm{px}$. Go to Filter $>$ Distort $>$ Polar Coordinates and pick Rectangular to Polar. Hit Cmd/Ctrl+U, check 
Colorize, set Hue 211. Paint white over any lines that show through.

\section{Convert photo to cartoon with Paint.net}

Paint.net is a fee photo editor for Windows, depending on the .Net framework. As one of the best alternatives for
Microsoft Paints, Paint.net offers more effects and features, including make cartoon photos with personal images.

Step 1: Import photo

Step 2: Add effect

Step 3: Simulate cartoon

Step 4: Fill background

Then save the paint to local disk.

\section{Convert photo to cartoon using Adobe illustrator}

Step 1: Get Yourself a Picture and Upload to Adobe Illustrator

Choose your favorite picture and upload it to Illustrator. Select a large enough picture so that you will be able to draw over it easily. This program is vector based, that means you will be able to scale the final artwork without losing its form. It will always look great if you want to make it larger or smaller.

\begin{tabular}{|c|c|c|c|c|}
\hline $\begin{array}{l}\text { Year } \\
\text { of The } \\
\text { Paper }\end{array}$ & $\begin{array}{c}\text { Title of the } \\
\text { Paper }\end{array}$ & Methodology Used & Advantage & Limitation \\
\hline 2015 & $\begin{array}{l}\text { A Neural } \\
\text { Algorithm of } \\
\text { Artistic Style } \\
\text { (Leon A. Gatys, } \\
\text { Alexander S. } \\
\text { Ecker, Matthias } \\
\text { Bethge) }\end{array}$ & $\begin{array}{l}\text { An artificial system based on a } \\
\text { Deep Neural Network that } \\
\text { creates artistic images of high } \\
\text { perceptual quality. we take } \\
\text { pre-trained neural network } \\
\text { and define a } 3 \text { Component loss } \\
\text { function that will enable us to } \\
\text { achieve our end goal of style } \\
\text { transfer and then optimize } \\
\text { over that loss function. }\end{array}$ & $\begin{array}{l}\text { First Ever Successful Attempt to } \\
\text { create Artistic style transfer using } \\
\text { Deep Neural Network. All previous } \\
\text { attempts/algorithms to achieve style } \\
\text { transfer had failed. The system uses } \\
\text { neural representations to separate } \\
\text { and recombine content and style of } \\
\text { arbitrary images, providing a neural } \\
\text { algorithm for the creation of artistic } \\
\text { images. }\end{array}$ & $\begin{array}{l}\text { While the Gatys } \\
\text { et al. method } \\
\text { produced } \\
\text { beautiful neural } \\
\text { style transfer } \\
\text { results, the } \\
\text { problem was that } \\
\text { it was quite slow. }\end{array}$ \\
\hline 2016 & $\begin{array}{l}\text { Perceptual Losses } \\
\text { for Real-Time } \\
\text { Style Transfer } \\
\text { and Super- } \\
\text { Resolution } \\
\text { (Justin Johnson, } \\
\text { Alexandre Alahi, } \\
\text { Fei-Fei Li) }\end{array}$ & $\begin{array}{l}\text { proposes the use of perceptual } \\
\text { loss functions for training } \\
\text { feed-forward networks for } \\
\text { image transformation tasks. a } \\
\text { feed-forward network is } \\
\text { trained to solve } \\
\text { the optimization problem } \\
\text { proposed by Gatys et al. in } \\
\text { real-time. }\end{array}$ & $\begin{array}{l}\text { Johnson et al. (2016) built on the } \\
\text { work of Gatys et al., proposing a } \\
\text { neural style transfer algorithm that is } \\
\text { up to three orders of magnitude } \\
\text { faster. The Johnson et al. method } \\
\text { frames neural style transfer as a } \\
\text { super-resolution-like problem based } \\
\text { on perceptual loss functions. }\end{array}$ & $\begin{array}{l}\text { While the } \\
\text { Johnson et al. } \\
\text { method is } \\
\text { certainly fast, the } \\
\text { biggest downside } \\
\text { is that you cannot } \\
\text { arbitrarily select } \\
\text { your style images } \\
\text { like you could in } \\
\text { the Gatys et al. } \\
\text { method. }\end{array}$ \\
\hline 2017 & $\begin{array}{l}\text { Instance } \\
\text { Normalization: } \\
\text { The Missing } \\
\text { Ingredient for } \\
\text { Fast Stylization } \\
\text { (Dmitry Ulyanov, } \\
\text { Andrea Vedaldi, } \\
\text { Victor } \\
\text { Lempitsky) }\end{array}$ & $\begin{array}{l}\text { The change is limited to } \\
\text { swapping batch normalization } \\
\text { with instance normalization, } \\
\text { and to apply the latter both at } \\
\text { training and testing times. The } \\
\text { resulting method can be used } \\
\text { to train high-performance } \\
\text { architectures for real-time } \\
\text { image generation. }\end{array}$ & $\begin{array}{l}\text { it was found that swapping batch } \\
\text { normalization for instance } \\
\text { normalization (and applying instance } \\
\text { normalization at both training and } \\
\text { testing) in Gatys et al. method, leads to } \\
\text { even faster real-time performance and } \\
\text { arguably more aesthetically pleasing } \\
\text { results as well. }\end{array}$ & None \\
\hline
\end{tabular}

Step2: Draw or Outline the Image

Now it's time to put your tracing skills to the test in adobe Illustrator.

Step1. Open a new layer.

Step2. Lock It.

Step3. Now use the pen tool to draw the outline of your original picture. At this point you can choose how detailed you want to be. A simple outline will work for starters.

Step3: Add Colors to You Picture

Now comes the exciting part where you can add color to the picture. You want to use shades of colors that is close to the original, unless of course you are looking for a different effect. An easy way to do this is to outline each color segment separately or its own layer. This will enable you to color each section and add your gradients.

Step4: Refine with Gradient Colors and Background Now you want to be a little but more detailed with the gradient tool to give your picture depth. You can play around with solid colors and gradients until it looks great. Adobe illustrator offers many options to create different effects. After Detailed analysis of above steps we realize above techniques/methods are not feasible or easy to use for a user with a simple requirement of applying a cartoon effect to his images. All the above methods are time and space consuming. Hence, we aim to build a website which provides easy to use interface to apply cartoon effects to the user.

\section{Review of Literature}




\section{Proposed System}

We propose to use neural style transfer which is a machine learning algorithm, which involves two images, first is the input image from the user and second is the style image which is used to apply the style on the input image. Following are the examples of images generated using neural style transfer

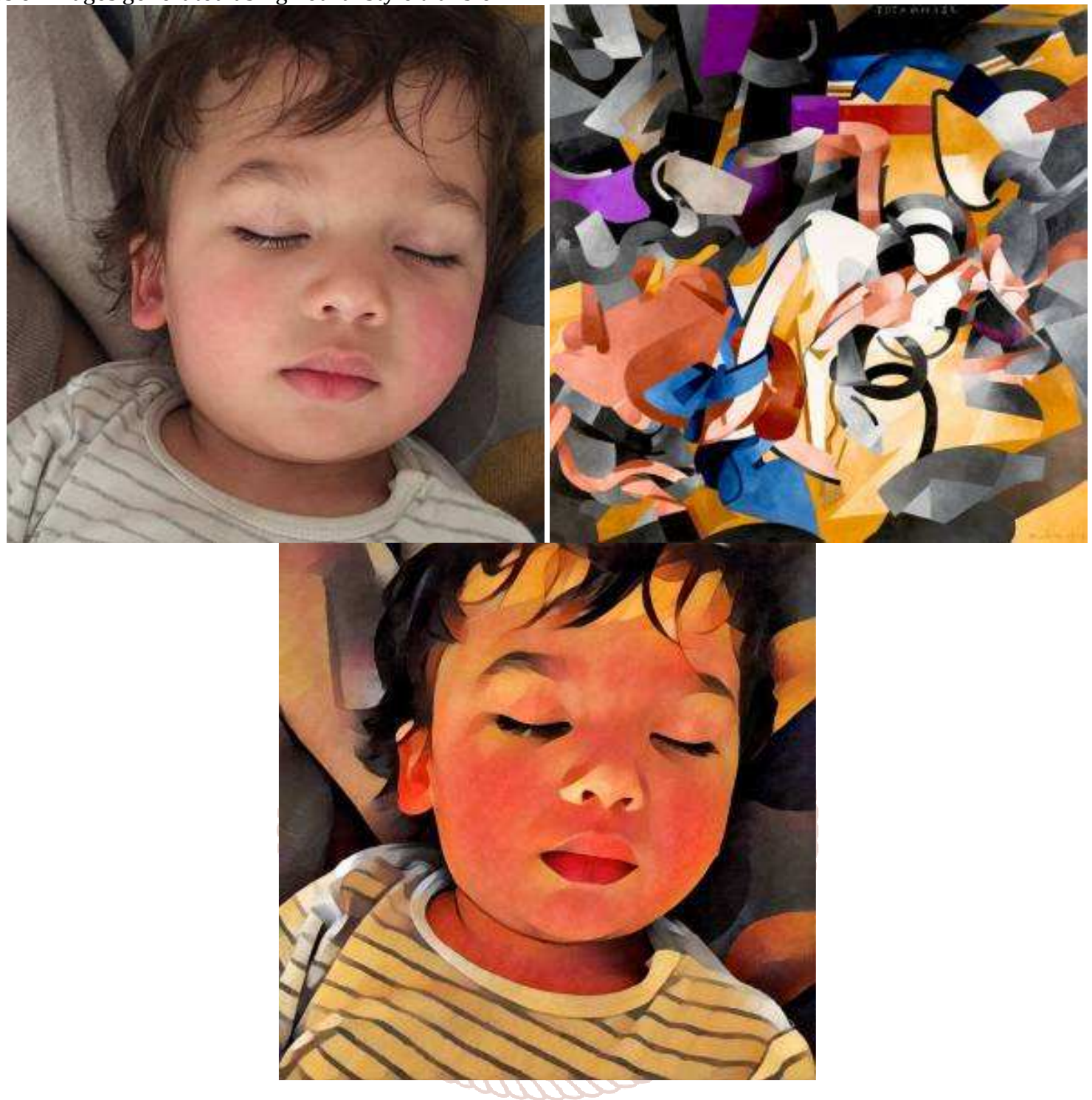

We propose to create a website, which consists of image upload functionality using which the user can upload his image, the uploaded image is then processed by server using Neural style transfer algorithm and the resulting image is presented to the user on the website. Which then user can download \& share. Neural fast style transfer is used by Apps such as https://deepart.io, Prisma, Artisto etc. We decided to choose this approach over traditional image filters (e.g. using image filters such as median \& bilateral filters to posterize an Image) as Neural fast style transfer is quite new and challenging technique which uses machine learning \& image processing to produce various styled images based on variety of input \& style images. The algorithm can be implemented in Python/JavaScript/Lua to perform neural style transfer. We will use Python to implement the backend and the front end of the website will be in HTML, CSS \& JS.

Basically, in Neural Style Transfer we have two images- style and content. We need to copy the style from the style image and apply it to the content image. By, style we basically mean, the patterns, the brushstrokes, etc. we will provide a set of style images which a user can use to apply different kinds of Cartoon like effects to his image.

Neural Style Transfer

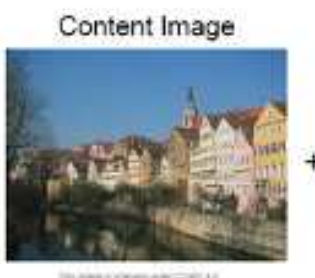

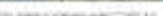

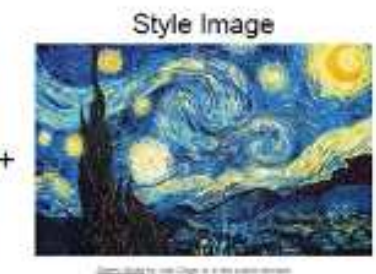

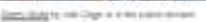

\section{Style Transfer!}

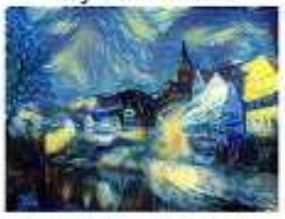




\section{Scope}

User will be provided with a set of pretrained style images to choose from. Based on the chosen style and the content image provided by the user, the Resulting image with cartoon like effect is generated by the program. The implementation is based on of the combination of Gatys' A Neural Algorithm of Artistic Style, Johnson's Perceptual Losses for Real-Time Style Transfer and Super-Resolution, and Ulyanov's Instance Normalization (all 3 papers mentioned above).

\section{Block Diagram}

Flow Diagram:

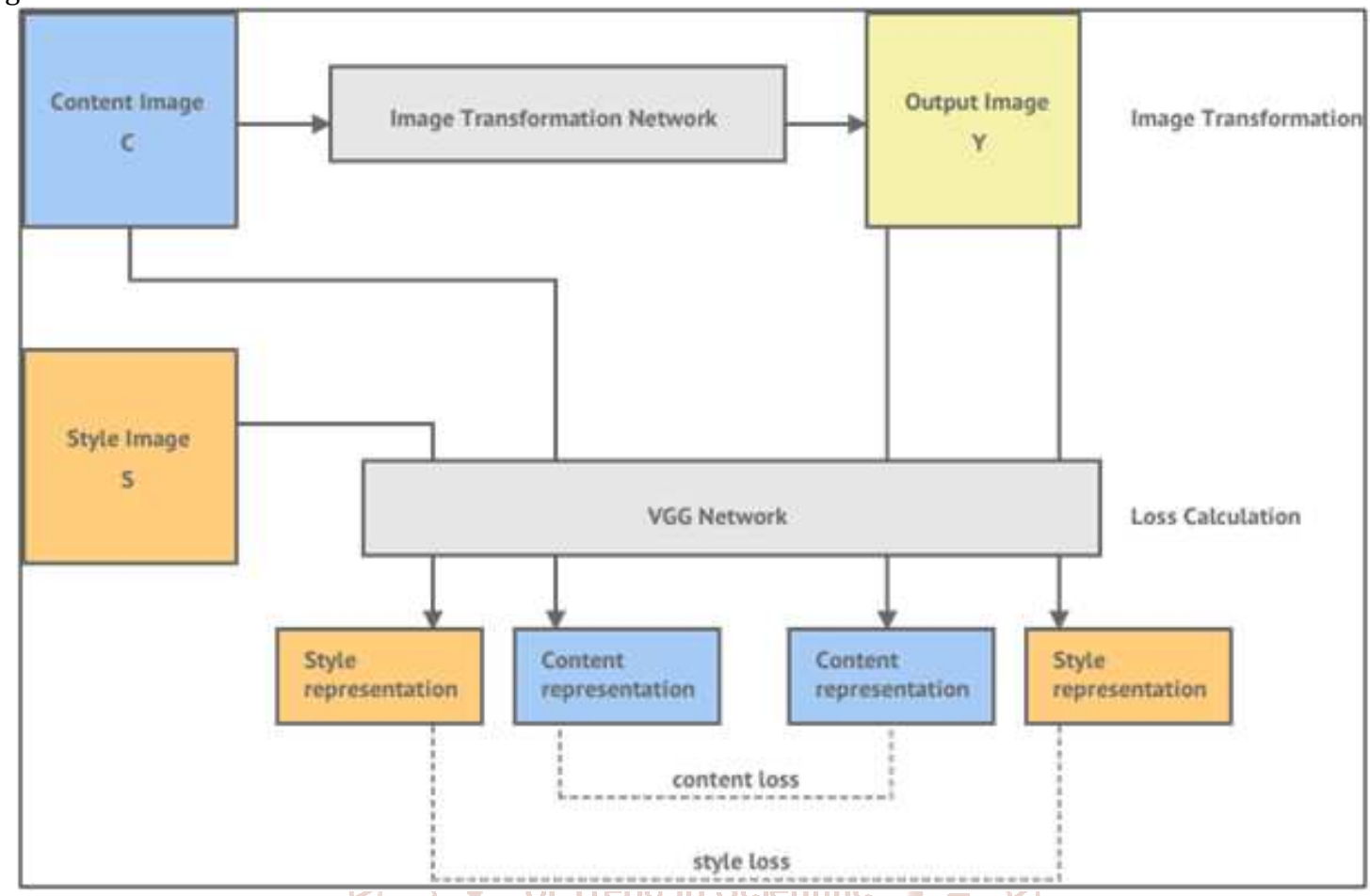

Process Diagram:

\section{Fast Style Transfer}

(1) Train a feedforward network for each style

(2) Use pretrained $\mathrm{CNN}$ to compute same losses as before

(3) After training, stylize images using a single forward pass

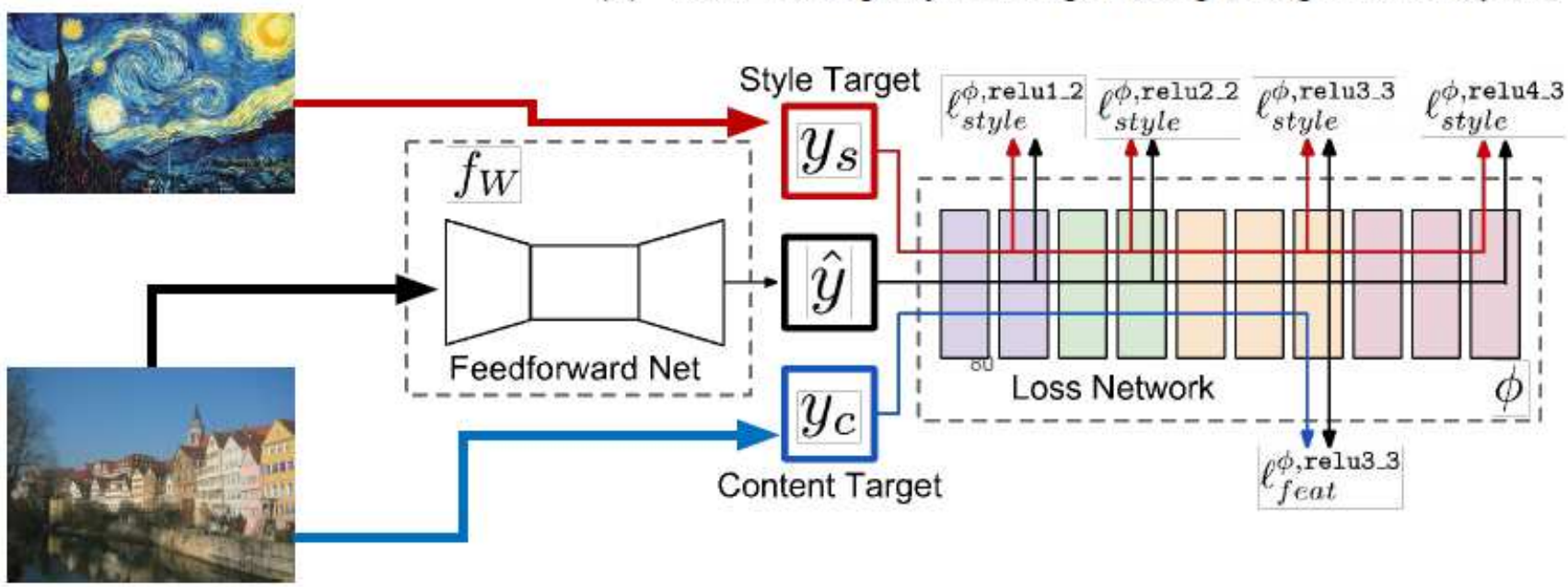

\section{Algorithm}

Our implementation uses TensorFlow to train a fast style transfer network. We use roughly the same transformation network as described in Justin Johnson et. al, except that batch normalization is replaced with Ulyanov's instance normalization. We use a loss function close to the one described in Gatys, using VGG19 instead of VGG16 and typically using "shallower" layers than in Johnson's implementation (e.g. we use relu1_1 rather than relu1_2). Empirically, this results in larger scale style features in transformations. 


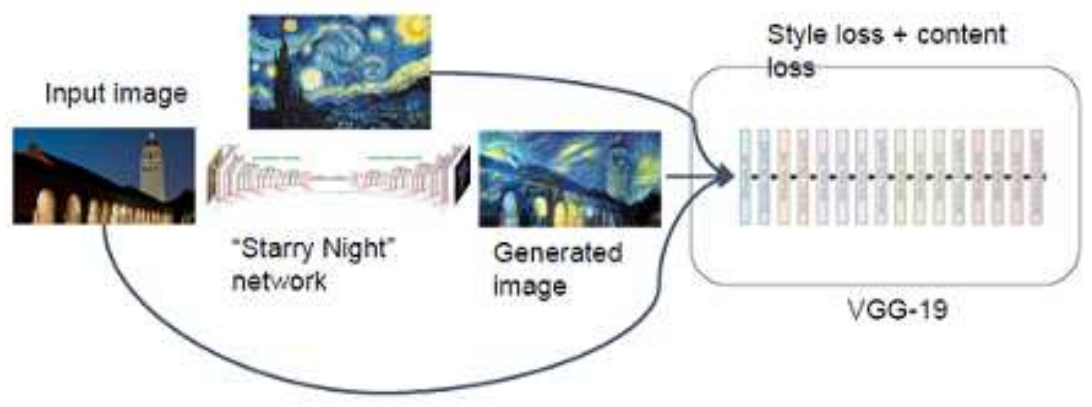

Train a layer-specific style transfer model

Step1. Train a feed forward neural network for each style

Step2. fetch the uploaded Content image(i.e. uploaded user image) \& trained feed forward network for the selected style image (i.e. style selected by user)

Step3. Calculate the Cost/Loss function of Generated Image G using

$\mathrm{f}(\mathrm{G})=\mathrm{f}_{\text {Content }}(\mathrm{C}, \mathrm{G})+\mathrm{f}_{\text {Style }}(\mathrm{S}, \mathrm{G})$

where $\mathrm{C}$ : Content Image \& S: Style Image

$\mathrm{f}_{\text {Content }}(\mathrm{C}, \mathrm{G})$ - Content Loss Function

$\mathrm{f}_{\text {Style }}(\mathrm{S}, \mathrm{G})$ - Style Loss Function

Step4. After minimizing the loss function $\mathrm{f}(\mathrm{G})$, Stylize the Image by single forward pass through image transformation network

Step5. Display Styled Image to the user

1. $H / W$ and $S / W$ requirement

Hardware Requirements:

$>$ Windows 10, 64 bits PC or 64 Bit Mac OS X High Sierra computers

$>$ Any CPU (Intel i5/i7/ Xeon recommended).

$>$ Nvidia GPUs (minimum 2GB Recommended)

>At least 8 GB RAM, 10 GB HDD Free Space.

Software Requirements:

$>$ Anaconda Framework for Python IDE \& Packages (Recommended), Jupyter notebook, Tensorflow machine Learning library

Programming Language: Python

\section{Challenges and Problem}

Training of networks for different style images is time consuming and requires lots of computation hardware(GPUs). Different content images may produce slightly different styled images. Precision of cartoon like effect entirely depends on type of content image provided.

\section{CONCLUSION:}

Thus we have shown that how image can be converted to cartoon. We also stated the examples on how image is converted to cartoon. Hardware and software requirements of image to cartoon conversion are also shown in this paper. The systematic working of image to cartoon conversion and respective algorithm and formulae is shown with neat diagram in this paper. Also we have stated challenges and problems one can face while cartoonifying the captured image. In this paper we have also discussed need and scope of cartoonifying the content image

\section{REFERENCES:}

[1] A Neural Algorithm of Artistic Style, 2016 - Leon A. Gatys, Alexander S. Ecker, Matthias Bethge
[2] Image Style Transfer Using Convolutional Neural Networks, 2016 - Leon A. Gatys, Alexander S. Ecker, Matthias Bethge

[3] Perceptual Losses for Real-Time Style Transfer and Super-Resolution, 2016 - Justin Johnson, Alexandre Alahi, Li Fei-Fei

[4] Precomputed Real-Time Texture Synthesis with Markovian Generative Adversarial Networks, 2016 Chuan Li, Michael Wand

[5] Texture networks: Feed-forward synthesis of textures and stylized images, 2016 - D. Ulyanov, V. Lebedev, A. Vedaldi, V. Lempitsky

[6] Demystifying Neural Style Transfer, 2017 - Yanghao Li, Naiyan Wang, Jiaying Liu, Xiaodi Hou

[7] A Learned Representation For Artistic Style, 2017 Vincent Dumoulin, Jonathon Shlens, Manjunath Kudlur

[8] Deep Photo Style Transfer, 2017 - Fujun Luan, Sylvain Paris, Eli Shechtman, Kavita Bala

[9] Neural Style Transfer: A Review, 2018 - Yongcheng Jing, Yezhou Yang, Zunlei Feng, Jingwen Ye, Yizhou Yu, Mingli Song

[10] StyleBank: An Explicit Representation for Neural Image Style Transfer, 2017 - Dongdong Chen, Lu Yuan, Jing Liao, Nenghai Yu, Gang Hua

[11] Conditional Fast Style Transfer Network, 2017 - Keiji Yanai, Ryosuke Tanno

[12] Characterizing and Improving Stability in Neural Style Transfer, 2017 - Agrim Gupta, Justin Johnson, Alexandre Alahi, Li Fei-Fei 